\title{
Towards Automatic Behavior Synthesis of a Coordinator Component for Context-Aware Mobile Applications
}

\author{
Laura M. Daniele, Luís Ferreira Pires, and Marten van Sinderen \\ Centre for Telematics and Information Technology \\ University of Twente \\ Enschede, the Netherlands \\ e-mail: \{l.m.daniele, l.ferreirapires, m.j.vansinderen\}@ewi.utwente.nl
}

\begin{abstract}
Although behavior modeling should play a central role in model-driven application development, it is still unclear how behavior modeling should be incorporated in model transformations. This paper presents an MDA-based approach that incorporates behavior modeling at the Platform-Independent Model (PIM) level of the design process of a specific category of applications, i.e., context-aware mobile applications. This approach includes two model transformations. The first transformation is a refinement of the (external) observable application behavior into a (internal) structured behavior based on a reference architecture. The second transformation is the synthesis of this structured behavior into the behavior of individual architecture components. This paper focuses on the second transformation and targets the behavior of a coordinator component that orchestrates the interactions between all the other components. We propose a state machines-based approach that can be used for synthesizing the coordinator component behavior in order to cope with synchronization and concurrency issues.
\end{abstract}

Keywords-behavior modeling; behavior synthesis: refinement; model-driven architecture; context-awareness; mobile applications

\section{INTRODUCTION}

Although the Model-Driven Architecture (MDA) guidelines $[13,18]$ prescribe that behavioral aspects should also be considered at the Platform-Independent Model (PIM) level of the application development process, current approaches mostly focus on the structural aspects and generation of code skeletons, and give less attention to the behavior of the modeled applications. Consequently, application behavior is largely overlooked at the PIM level [19], and behavioral aspects have to be incorporated later in the development process, by adding hand-written code as annotations to Platform-Specific Models (PSMs) or to implementation code skeletons. This paper presents an MDA-based approach that incorporates behavior modeling at the PIM level in the development of a specific category of applications, i.e., context-aware mobile applications.
Following the MDA principles, this approach is centered on models and transformations between these models. Particularly, our PIM level is decomposed in three models and two model transformations. The first model (service specification) defines the external observable behavior of the application, the second model (service design refined model) refines this external behavior in a structured behavior according to a reference architecture for contextaware mobile applications, and the third model (service design component model) assigns this structured refined behavior to concrete architecture components. This paper focuses on this second transformation step and proposes a formalism that can be used to support behavior synthesis of our components, especially of a coordinator component that orchestrates the interactions between all the other components. The paper also discusses how synchronization and concurrency issues in the behavior of the coordinator component can be handled in this transformation.

The structure of the paper is the following: Section II presents our reference architecture for context-aware mobile applications, Section III presents an overview of models and transformations of our MDA-based approach, Section IV illustrates the second transformation step of our approach with an example, Section V presents a state machines-based approach to support this second transformation step, Section VI discusses a possible alternative to this approach, and Section VII discusses some related work. Finally, Section VIII presents our conclusions and identifies topics for future work.

\section{REFERENCE ARCHITECTURE}

The reference architecture we have considered in our approach targets a specific family of applications, i.e., context-aware mobile applications. These are intelligent applications capable to sense changes in the user's environment and consequently adjust their behavior in order to provide relevant functionality to their users anywhere and at any time. 


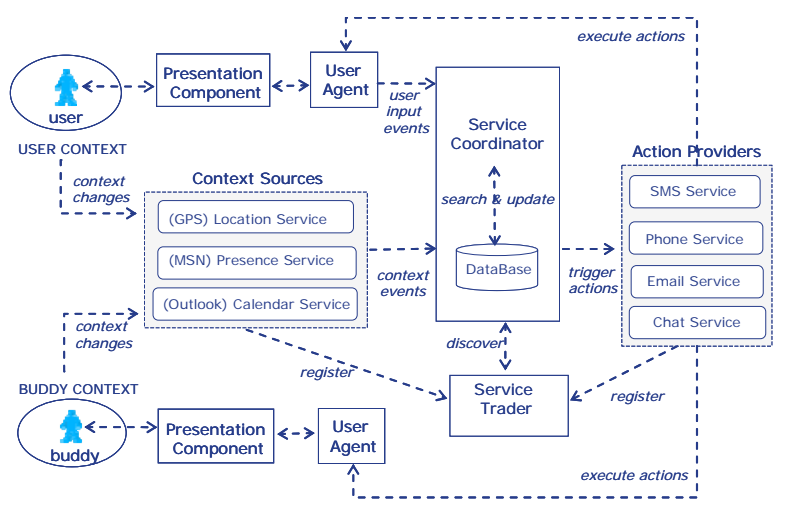

Figure 1. Reference architecture for context-aware mobile applications.

Fig. 1 shows our reference architecture, which has been defined and applied in the A-MUSE project to realize the Live Contacts case study [11,15]. Live Contacts consists of a context-aware mobile application that runs on Pocket PC phones, Smartphones and desktop PCs and allows its users to contact the right person, at the right time, at the right place, via the right communication channel.

Fig. 1 shows a single user instance interacting with the system and a buddy of this user. The presentation component takes care of the interactions with the end-user. The user agent (one for each user) acts on behalf of the user with the presentation component to obtain user input and present user output, and provides the service coordinator with user input events. The service coordinator takes care of orchestrating the other components, searching and updating a database, which contains information about users (e.g., name, password, preferred contact means and list of buddies). To simplify the discussion without loss of generality, we assume that there is one service coordinator and one database. The service coordinator also interacts with context sources and action providers.

The context sources sense changes in the user's context and provides the service coordinator with context events. Fig. 1 shows the (GPS) location service that provides information about a user's current location, the (MSN) presence service that provides indications whether users registered in the Live Contacts application are available online in the network, and the (Outlook) calendar service that provides calendar information. In this example we assume that there is one (GPS) location service, one (MSN) presence service and one (Outlook) calendar service for each user agent. These services are registered in the service trader.

The action providers are responsible for performing actions triggered by the service coordinator. Actions represent application reactions to user input events and context events. Fig. 1 shows an SMS service, phone service, e-mail service and chat service, which enable a user to communicate with buddies through, respectively, sending messages, making a phone call, sending e-mails or chatting. We assume that there is one of these services for each user agent. These services are registered in the service trader, which registers all the available context sources and action providers in order to allow the coordinator to discover and invoke them.

Although the architecture in Fig. 1 has been defined for the Live Contacts application, it is general enough to be reused for other context-aware mobile applications by simply redefining some application-specific components, such as context sources and action providers. Moreover, the use of this architecture for context-aware mobile applications does not limit our MDA-based approach to this family of applications, since the same models and model transformations can be defined and applied to other categories of applications based on different reference architectures.

\section{MDA-BASED APPROACH}

Fig. 2 shows our MDA-based approach for the behavior modeling of applications. Fig. 2 shows that the service specification (SS) is the highest level of abstraction of this approach. The SS describes a context-aware mobile application as a monolithic behavior from an external perspective. At this level, we specify the functionality that our application offers to its user and we do not consider any structural detail, i.e., we abstract from application internal components. The actions and causality relations at the SS level are too abstract to be directly realized by platformspecific technologies. Therefore, transformation $\mathrm{T}_{1}$ refines the SS into the service design refined model (SDRM), in which actions and causality relations are decomposed into more detailed actions and causality relations that can be mapped to the reference architecture of Fig. 1. In the SDRM, we have been able to identify sequences of actions that recur in the refinement of several application functions. We call these sequences of actions interaction patterns, which we have defined in [2] as "recurring sequence of actions performed by two or more interacting components defined from the internal perspective of a service”.

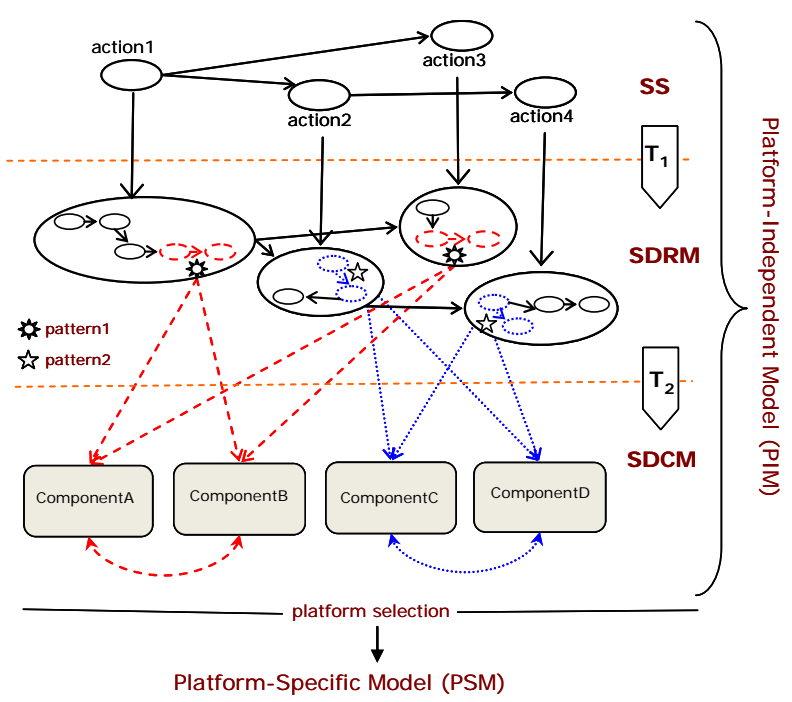

Figure 2. MDA-based approach. 
Fig. 2 shows two interaction patterns. The first interaction pattern, which we have marked with the " symbol, recurs in the refinement of actions 1 and 3 . The second interaction pattern, which we have marked with the “»” symbol, recurs in the refinement of actions 2 and 4 . Since we aim at synthesizing the behavior of individual components of our reference architecture, the last step of our approach consists of assigning the interaction patterns of the SDRM to the corresponding components that realize these patterns. This assignment (transformation $\mathrm{T}_{2}$ in Fig. 2) results in the service design component model (SDCM). Fig. 2 also shows that the interaction pattern marked with the " symbol is assigned with dashed arrows to the corresponding components (components $\mathrm{A}$ and $\mathrm{B}$ ), and the interaction pattern marked with the “纺” symbol is assigned with dotted arrows to components $\mathrm{C}$ and $\mathrm{D}$.

The SDCM provides the input for the platform-specific model (PSM) level without imposing any specific implementation choices. Therefore, it is in principle possible to use different middleware technologies to realize the platform-specific service design. For example, our coordinator component can be implemented as a BPEL (Business Process Execution Language) process, action providers as web services, and the service trader as a UDDI registry.

Our previous work [3] focuses on transformation $T_{1}$. This paper focuses on transformation $\mathrm{T}_{2}$.

\section{TRANSFORMATION $\mathrm{T}_{2}$}

This section illustrates transformation $\mathrm{T}_{2}$ from SDRM to SDCM for the Live Contacts case study. This transformation takes as input a source model in the AMUSE Domain Specific Language (DSL) and produces as output a target model in the Interaction System Design Language (ISDL) [9]. The A-MUSE DSL and ISDL are general purpose languages that allow us to model application behavioral aspects in terms of causality relations between interactions without constraining the internal implementation of the modeled application. Our approach is not restricted to any specific modeling language and in this work the A-MUSE DSL and ISDL are only vehicles to reach our purposes. Fig. 3 and Fig. 4 show excerpts of source and target models for transformation $\mathrm{T}_{2}$, respectively.

\section{A. Source Model}

Fig. 3 shows an example of SDRM with two functions: (i) remove buddy in Fig. 3(a), which is an instance of the user request with acceptance or rejection response pattern, and (ii) proximity in Fig. 3(b), which is an instance of the context event with alert pattern. The user request with acceptance or rejection response pattern consists of a user request followed by confirmation whether the required task has been successfully performed or not. The context event with alert pattern consists of a notification of a context event. In order to avoid clogging the figure, we have not included the status information handled in the SDRM. However, we have marked each action in Fig. 3 with a label that represents an interaction between two components and the direction of this interaction.

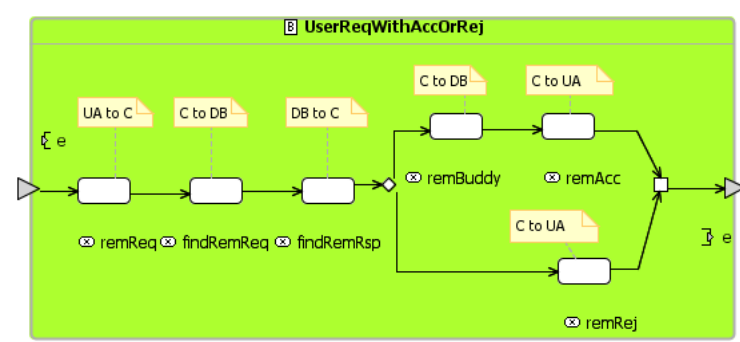

(a)

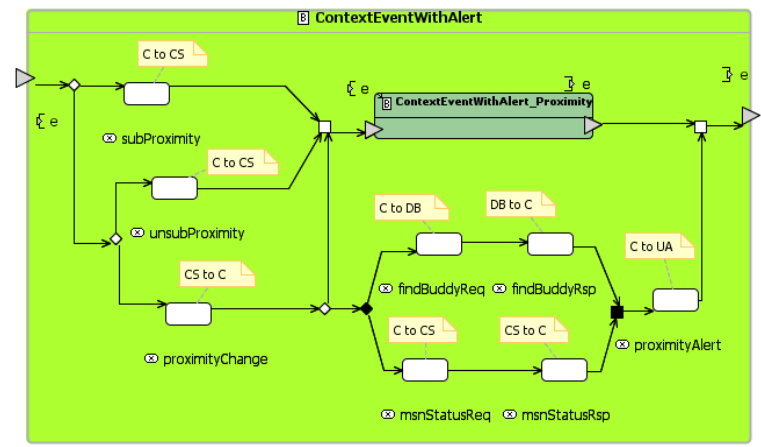

(b)

Figure 3. Example: service design refined model (SDRM).

Fig. 3(a) shows that the user request to remove a buddy from his/her list (remReq) arrives from the user agent (UA) to the coordinator $(\mathrm{C})$. The coordinator checks the database (DB) to determine whether the buddy is included in the buddy list of the user (findRemReq and findRemRsp). If this is the case, the coordinator removes the buddy from the list (remBuddy) and sends a positive response to the user agent (remAcc). If the buddy is not in the list, the coordinator sends a negative response to the user agent (remRej).

Fig. 3(b) shows that in order to notify the user of the occurrence of a proximity event, the coordinator has first to subscribe in the context sources (CS) for that particular event (subProximity). Upon the occurrence of the proximity event, i.e., when a buddy is nearby the user, the context source notifies the coordinator (proximityChange). While retrieving the buddy name from the database (findBuddyRea and findBuddyRsp), the coordinator requests to the context sources the current MSN status of the buddy (msnStatusReq and msnStatusRsp) in order to check if this value is "online". If this is the case, the coordinator generates an alert to the user agent (proximityAlert). Any time after subscribing for the proximity event, the coordinator can also unsubscribe (unsubProximity) if it is not interested any more in getting proximity notifications for a certain user's buddy.

In the complete SDRM of the Live Contacts application, we have been able to identify a library of recurring sequences of interactions between components (interaction patterns). Particularly, we have identified two types of interaction patterns, namely basic and composite patterns. Basic interaction patterns occur between two interacting components. An example of basic interaction patterns is the sequence findRemReq and findRemRsp in Fig. 3(a), which we call search pattern, and involves interactions between the 
coordinator (C) and the database (DB). Composite interaction patterns occur between more than two components and consist of combinations of basic patterns. Examples of composite interaction patterns are the remove buddy functionality in Fig. 3(a), and the refinement of the proximity functionality in Fig. 3(b). From our experience with the Live Contacts application we have defined a library of basic and composite interaction patterns that cover the functionality of context-aware mobile applications that comply with the reference architecture of Fig. 1.

\section{B. Target Model}

Transformation $\mathrm{T}_{2}$ takes the SDRM of Fig. 3 as source model and generates as target model an SDCM that represents the behavior of individual components. Fig. 4 depicts an example of SDCM in ISDL, which allows the specification of behavioral aspects of interacting components. Fig. 4 shows the assignment of the user request with acceptance or rejection composite pattern of Fig. 3(a) to components. The three involved components are the user agent (UA), the coordinator (C) and the database (DB). Dashed lines in Fig. 4 indicate the assignment of basic patterns to these components.

The component model of Fig. 4 has been obtained manually by assigning the interaction patterns to components that realize these patterns. Since the interaction patterns explicitly specify which components perform a certain action, the mapping of these patterns to the corresponding components is straightforward. However, synchronization and concurrency issues of interacting components are not considered in the mapping. These issues have to be addressed separately in order to generate service design component models that are correct and consistent with respect to the original application behavior.

For example, consider the behavior of the coordinator component in Fig. 3. The coordinator has to schedule somehow the execution of the composite interaction patterns in Fig. 3(a) and Fig. 3(b). Actually, the removal of a buddy from the contact list of the user implies that that user is not any more interested in receiving proximity alerts about the removed buddy. Therefore, after the removal of a buddy (remBuddy), the coordinator has to unsubscribe the proximity event for that buddy (unsubProximity). This interaction is not explicitly represented in the SDRM of Fig. 3, where the two execution threads for the buddy removal and the proximity event appear to be independent.

In addition to these functional aspects, the model of Fig. 3 considers only one user instance interacting with the system. In reality, the coordinator has to handle multiple user instances.

Therefore, in order to synthesize the behavior of our coordinator component we need formalisms that support two issues: (a) synchronization and concurrency within a single user instance, and (b) synchronization and concurrency of multiple user instances. We have identified a possible approach to support these aspects. We present a possible solution for both issues (a) and (b).

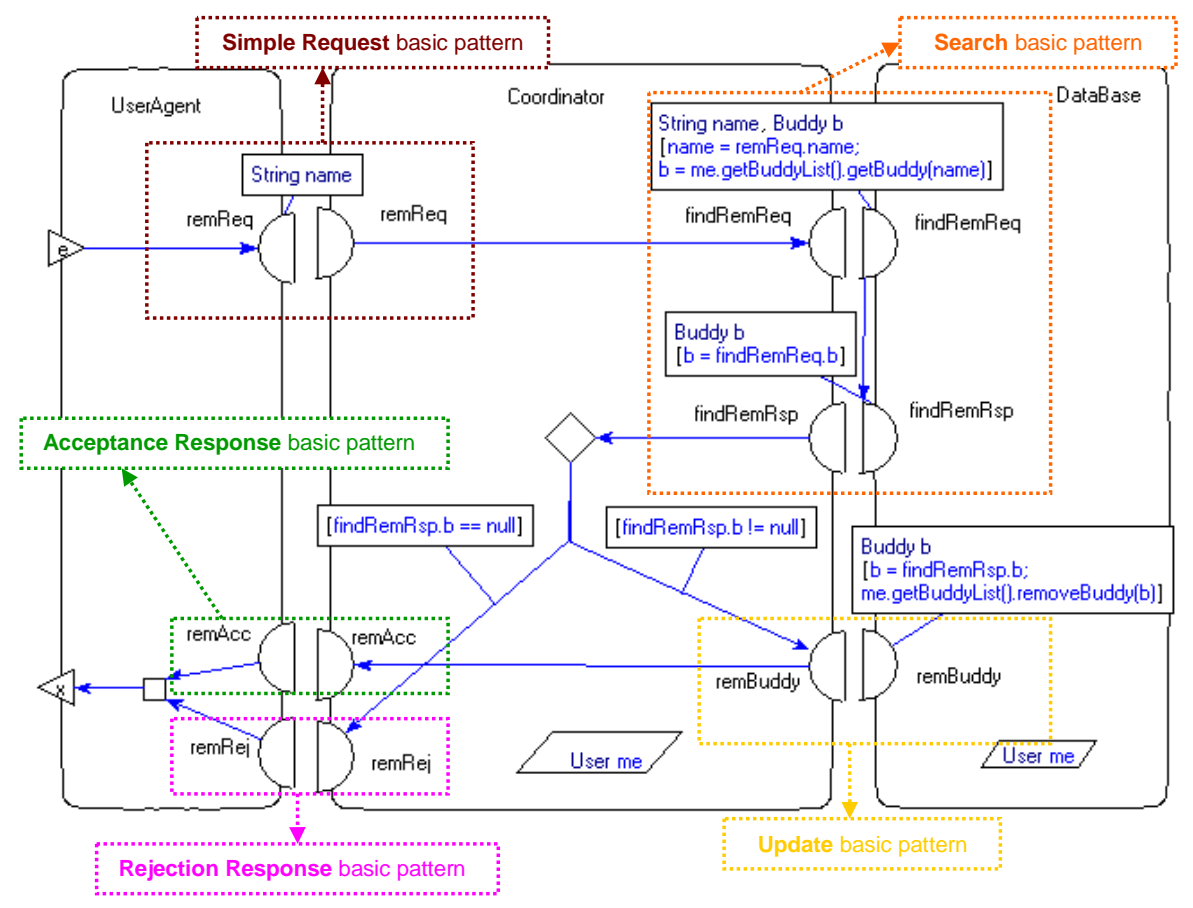

Figure 4. Example: service design component model (SDCM). 


\section{StATE MACHINES-BASEd APPROACH}

The approach for behavior synthesis we propose in this paper is based on state machines. State machines represent models consisting of states, transitions between these states, and actions. These models are suitable to represent behavioral aspects of applications, particularly for the specification of behaviors that are assigned to concrete components, such as our coordinator component.

We have based our state machines approach on the work presented in [16], where two types of behavior model synthesis techniques are analyzed: synthesis from properties and from scenarios. A behavior model synthesized from properties provides an upper bound of the modeled application, since it includes all possible acceptable behaviors of the application that do not violate those properties. However, it may not be necessary and advisable to model such a large set of behaviors (all these behaviors are possible, but not all of them are required). In contrast, a behavior model synthesized from scenarios provides a lower bound of the modeled application, since it includes a limited set of example behaviors that the application can assume. However, this set may considerably grow in case the scenario is extended (these example behaviors are required, but there are other possible behaviors that have not been considered yet). Therefore, [16] suggests that a comprehensive behavior model should be synthesized both from properties and scenarios. Traditional state machines models, such as Labeled Transition Systems (LTSs), cannot capture this middle ground between properties and scenarios, since LTSs do not support the distinction between required and possible behaviors. Therefore, a formalism based on Modal Transition Systems (MTSs) is proposed in [16], which allows one to distinguish possible from required behavior, preserving the original properties and scenario, and also supporting elicitation of new properties and scenarios. The following definitions provide some background on LTSs and MTSs. More details can be found in [16].

Definition 1 (Labeled Transition Systems) Let States be a universal set of states, and Act be a universal set of observable action labels. An LTS is a tuple $L=\left(S, A, \Delta, s_{0}\right)$, where $S \subseteq$ States is a finite set of states, $A \subseteq A c t$ is a set of labels, $\Delta(S \times A \times S)$ is a transition relation, and $s_{0} \in S$ is the initial state.

Definition 2 (Modal Transition Systems) An MTS $M$ is a structure $\left(S, A, \Delta^{r}, \Delta^{p}, s_{0}\right)$, where $\Delta^{r} \subseteq \Delta^{p},\left(S, A, \Delta^{r}, s_{0}\right)$ is an LTS representing required transitions of the system and $\left(S, A, \Delta^{p}, S_{0}\right)$ is an LTS representing possible (but not necessarily required) transitions.

Consider issue (a) identified in Section IV.B, i.e., how the coordinator can handle synchronization and concurrency within a single user instance interacting with the application. Fig. 5 depicts a possible solution that synthesizes the coordinator behavior from both properties and scenario.

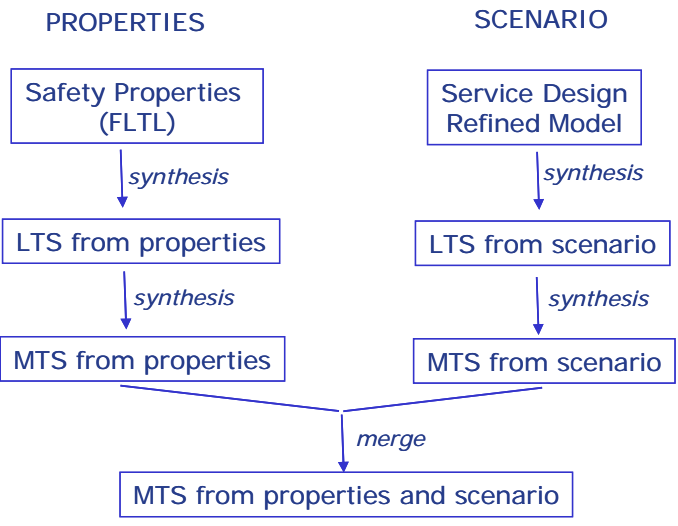

Figure 5. Transformation $\mathrm{T}_{2}$ : state machines-based approach..

Our scenario is the SDRM of Fig. 3. This scenario represents sequences of interactions between some components, i.e., the user agent, coordinator, database and context sources. We are interested in the behavior of the coordinator component. Using an adaptation of the technique described in [17], our approach synthesizes an LTS from the perspective of the coordinator. Fig. 6 shows an example of this LTS for the scenario in Fig. 3.

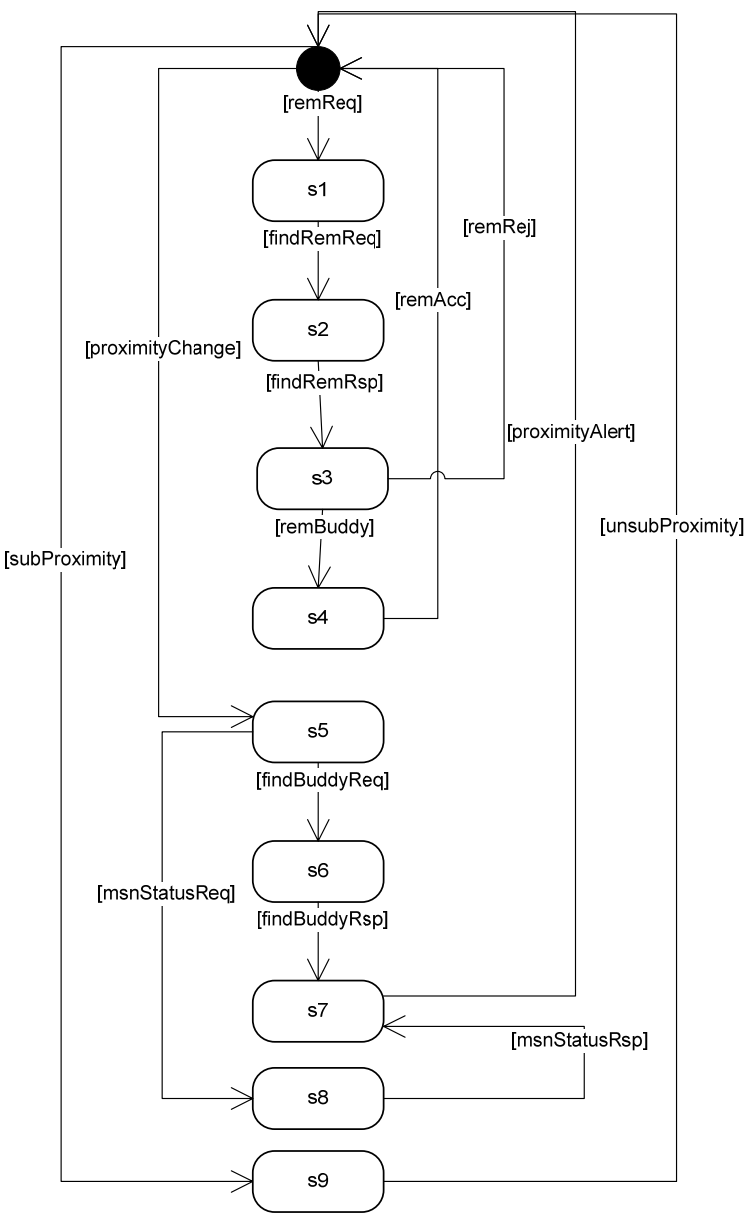

Figure 6. LTS from scenario. 


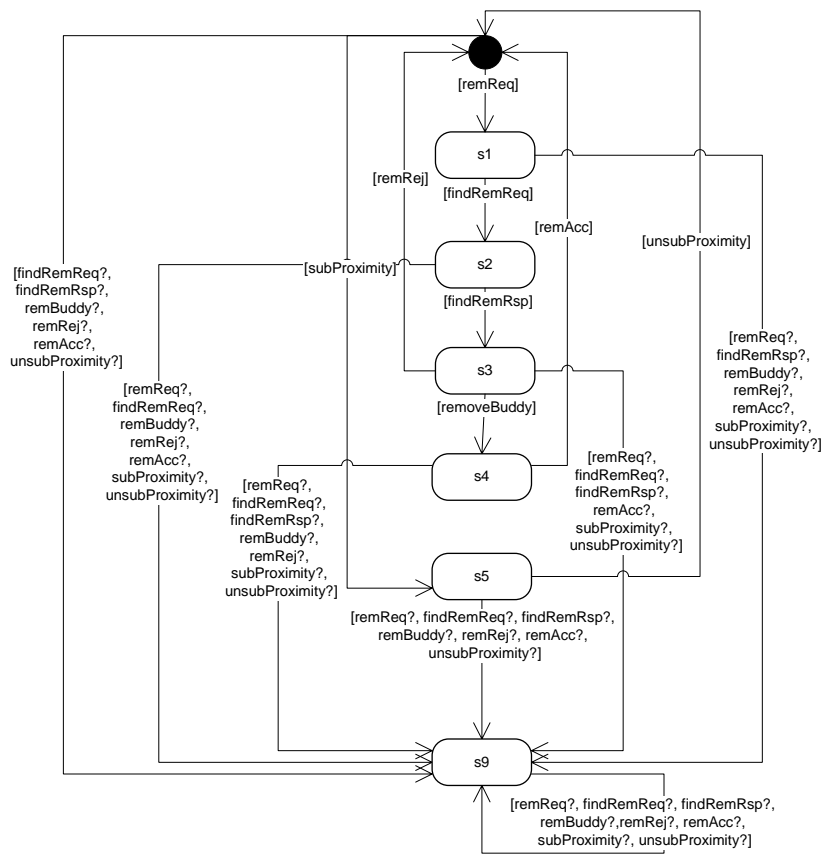

Figure 7. MTS from scenario.

Using the algorithm described in [16], we synthesized the LTS of Fig. 6 in an MTS, which is shown in Fig. 7. The MTS in Fig. 7 distinguishes possible from required transitions. Possible transitions are denoted with a question mark following the label. To simplify the example without loss of clarity, states $s 6$ to $s 8$ in Fig. 6 have been removed in Fig. 7.

The scenario represented by our SDRM does not cover relations between different execution threads (interaction patterns), such as the relation between the remove buddy and proximity instances, in which the coordinator has to unsubscribe the proximity event for a buddy (unsubProximity) as soon as this buddy has been removed from the contact list of the user (remBuddy). We can represent such relation as a safety property. Safety properties are those that specify that "nothing bad can happen". These properties can be expressed, for example, by using Fluent Temporal Logic (FLTL) as recommended in $[17,7]$. The FLTL property for the interaction between remBuddy and unsubProximity is the following:

$$
\mathbf{P}=\mathbf{G}(\text { remBuddy } \Rightarrow \mathbf{X} \text { unsubProximity })
$$

This formalization of $\mathrm{P}$ states that if remBuddy occurs, then the next $(\mathbf{X})$ event to occur is unsubProximity. An LTS can be generated from the safety property $\mathrm{P}$, as shown in Fig. 8(a), and this LTS can be synthesized in an MTS, as shown in Fig. 8(b). The state s-1 in Fig. 8(a) represents an error state and corresponds to undesired behaviors [16]. This state has been removed in the MTS of Fig. 8(b).

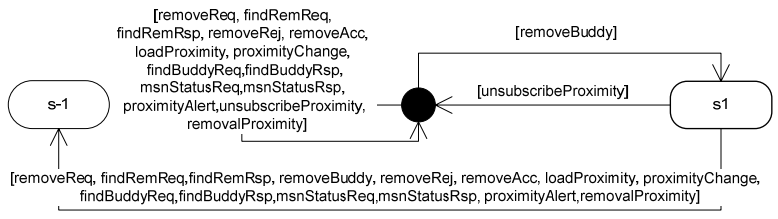

(a)

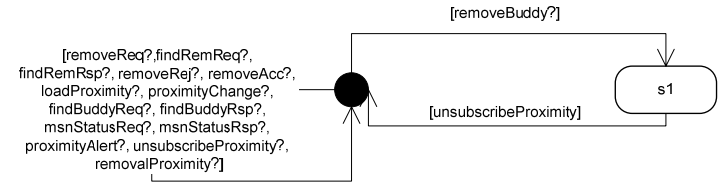

(b)

Figure 8. LTS and MTS from property P.

The two MTSs synthesized from properties and scenarios can be finally merged in one MTS, as is shown in Fig. 9. Reference [16] demonstrates that the merged MTS preserves the original properties and scenario. In this way, we can guarantee correctness and consistency to the original behavior through our model transformations.

In our example we have used only the property $\mathrm{P}=$ $\mathbf{G}($ remBuddy $\Rightarrow \mathbf{X}$ unsubProximity). However, additional properties can be defined to represent explicitly other relations between interaction patterns. By adding new properties, we can in principle reduce the possible transitions in Fig. 9 until there are only required transitions. In this case, we obtain an LTS. In practice, it may not be necessary to refine the MTS into a single LTS, since the designer may explicitly decide to leave some behavioral choices open further down in the development process.

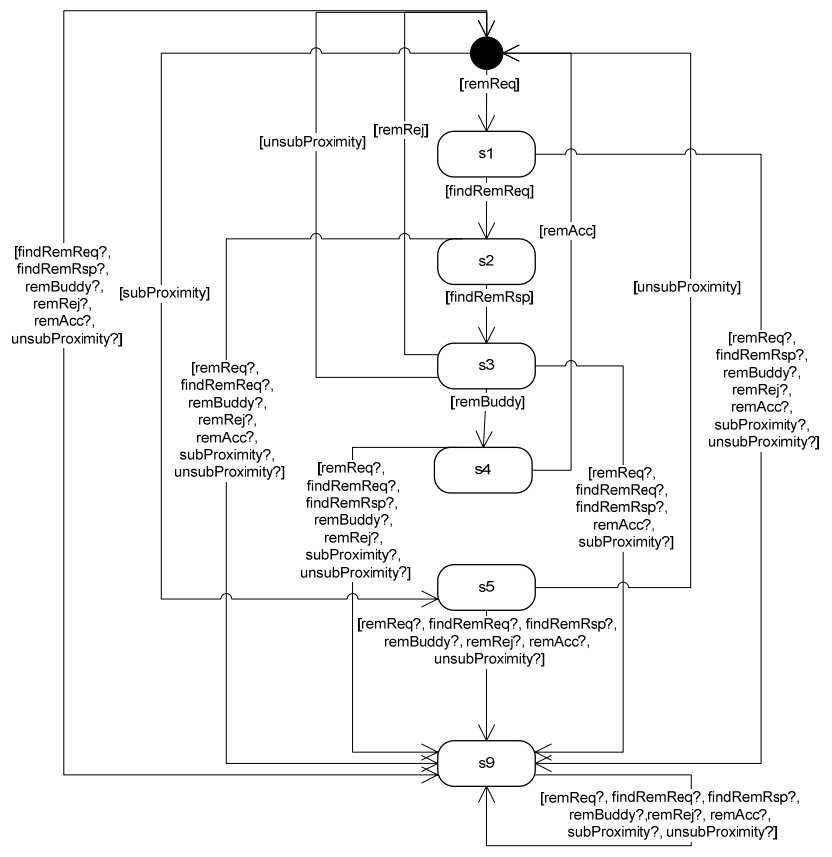

Figure 9. MTS from properties and scenario. 
Consider now issue (b) mentioned in Section IV.B, i.e., how the coordinator can handle synchronization and concurrency of multiple user instances interacting with the application. This issue can be handled by using the same synthesis approach from properties and scenario of Fig. 5. The only difference is that the scenario should be represented with a different language than A-MUSE DSL, since the SDRM of Fig. 3 does not give support to the representation of multiple user instances. An alternative consists of defining this scenario with a sequence diagram that considers $\mathrm{N}$ users interacting with the coordinator. The safety properties would represent interactions between different user instances that cannot be captured in the sequence diagram described by the scenario.

The two MTSs obtained from issues (a) and (b) should be finally merged in one MTS. This MTS represents the final SDCM of the coordinator and correctly handles both multiple user instances interacting with the system and different execution threads within each of these user instances. This SDCM, which is platform-independent, should be taken as input at the PSM level in order to realize the coordinator with specific target technologies. A suitable technique to derive a platform-specific model can be the state pattern, which is a behavioral software design pattern that allows a systematic translation of state machine models to object-oriented programming code, such as Java code.

\section{DISCUSSION}

Some work in the literature $[4,14]$ attempts to develop transformations from platform-independent business process models to implementations in terms of BPEL. Since state machines are not suitable to be realized by BPEL implementations, an alternative approach for behavior synthesis of the coordinator component should also be considered. Such an approach should be based on process models. These models are suitable to represent behavioral aspects of applications in terms of process flow relationships, such as sequence, choice, iteration and concurrency.

The first step of process modeling should be the mapping of the SDRM of Fig. 3 into a process model of the coordinator. This model should consider both issues (a) and (b) of Section IV.B, i.e., respectively, the handling of internal dependencies within a single user instance interacting with the application, and the handling of multiple user instances running at the same time in the application. Several techniques can be used to model processes, such as, for example, UML 2 activity diagrams and Petri Nets. Both these techniques offer graphical notation for representing stepwise processes with support for choice, iteration and concurrency.

Once an SDRM process model of the coordinator has been defined, the second step should be the synthesis of the SDCM. Since this model is platform-independent, it should be represented in a language that is independent from both modeling languages and implementation languages. A suitable language for this purpose is ISDL [14]. When using ISDL, the SDCM would look like the example depicted in Fig. 4. Another suitable language to represent the service design component model is the CBPL (Common Behavioral Patterns Language) presented in [4]. CBPL is a pattern language used to represent constructs of implementation languages derived from structures of modeling languages in a platform-independent way. Both ISDL and CBPL (platform-independent) process models can be mapped to BPEL $[4,14]$.

\section{RELATED WORK}

We briefly discuss here some related work. Considerable effort has been spent on model transformations from PIMs to PSMs in several application domains. For example, in [10], a formal MDA approach for the development of mobile health systems is discussed. A model-driven approach for the development of access control policies for distributed systems is presented in [6]. In [5], an MDA approach to implement personal Information Retrieval (IR) processes is proposed. In contrast to our approach, these approaches do not consider behavior refinement and synthesis at the PIM level and directly start the development process by defining a platform-independent model of the architecture that implements the application, which roughly corresponds to our SDRM. We believe that behavior refinements and synthesis at the PIM level are the major novelties of our approach.

We used the concept of interaction patterns to identify recurring sequences of actions performed by two or more interacting components defined from the internal perspective of a service. This is a novel concept that differs from the common use of this term in the literature [8]. A concept rather similar to our concept of interaction pattern is presented in [1], where so called service interaction patterns are identified in order to cover multilateral, competing, atomic and causally related interactions. However, the service interaction patterns in [1] are used in benchmarking in order to identify platform-specific implementation issues, for example, in case BPEL is used. In contrast, our interaction patterns are related to the platform-independent level and are used to refine service specifications that are too abstract to be directly realized by application components. BPEL is a possible platform-specific technology to implement our interaction patterns. However, they can also be implemented with other platform-specific solutions, such as, for example, Java code.

The approach to transform service design refined models into the service design component models based on state machines presented in this paper is inspired by the work presented in [16]. In this work, an automated technique for behavior synthesis from properties and scenarios using Modal Transition Systems (MTSs) is proposed. A novel contribution of this paper is its attempt to incorporate this comprehensive and detailed technique for behavior model synthesis into an MDA-based approach that decomposes systematically and consistently the application design process in several steps with different degree of abstraction and platform-independence. 


\section{CONCLUSIONS}

In this paper, we presented an MDA-based approach for the behavior modeling of context-aware mobile applications. This approach integrates behavioral aspects of the modeled applications at the PIM level, resulting in three models and two model transformations.

The general contribution of our approach consists of applying model transformations that address behavior correctness issues systematically throughout the design process, from abstract design towards implementation. In our investigation, we first apply these transformations manually in order to learn how they could be automated. Afterwards, we use the acquired knowledge to select existing tool, or eventually to create new tools suitable to automate these transformations. The specific contribution of this paper is on transformation $\mathrm{T}_{2}$, namely on existing formalisms that are suitable to handle concurrency and synchronization issues of interacting components. We proposed a formalism based on LTS, MTS and safety properties. We also discussed some initial ideas about an alternative formalism based on process models. Transformation $\mathrm{T}_{2}$ has not been automated yet, but this paper is a step towards its automation. The proposed formalism gives a suitable theoretical foundation for $\mathrm{T}_{2}$ and our future work consists of investigating suitable tool support for this formalism.

Our previous work focused on the transformation $T_{1}$ of our approach, namely the transformation from SS to SDRM. In [3], we discussed the automation of this transformation with the Medini QVT tool [14], which allowed us to define transformation rules in the QVT Relation language defined by OMG.

\section{ACKNOWLEDGMENT}

This work is part of the Freeband A-MUSE Project (http://a-muse.freeband.nl). Freeband is sponsored by the Dutch government under contract BSIK 03025.

\section{REFERENCES}

[1] A. Barros, M. Dumas, and H.M. ter Hofstede, "Service interaction patterns," Business Process Management, Lecture Notes in Computer Science, vol. 3649, Springer Verlag, 2005, pp. 302-318.

[2] L. Daniele, L. Ferreira Pires, and M. van Sinderen, "Interaction patterns for refining behavior specifications of context-aware mobile services," Proc. of the 4th Int'l Workshop on Model-Driven Enterprise Information Systems (MDEIS 2008), INSTICC Press, 2008, pp. 64-76.

[3] L. Daniele, L. Ferreira Pires, and M. van Sinderen, "An MDA-based approach for behavior modeling of context-aware mobile Applications," Proc. of the 5th European Conf. on Model Driven architecture-Foundations and Applications (ECMDA-FA 2009), Lecture Notes in Computer Science, vol. 5562, Springer Verlag, 2009, pp. 206-220.

[4] T. Dirgahayu, D. Quartel, and M. van Sinderen, "Development of transformations from business process models to implementations by reuse," Proc. of the 3th Int'l Workshop on Model-Driven Enterprise Information Systems (MDEIS 2007), INSTICC Press, 2007, pp. 4150 .

[5] S. M. Eissen, and B. Stein, "An MDA approach to implement personal IR tools,” 18th Int'l Conf. on Database and Expert Systems
Applications (DEXA 2007), IEEE Computer Society Press, 2007, pp 259-263.

[6] T. Fink, M. Koch, and K. Pauls, "An MDA approach to access control apecifications using MOF and UML profiles," First Int'l Workshop on Views on Designing Complex Architectures (VODCA 2004), Electronic Notes in Theoretical Computer Science, Vol. 142, 2006, pp 161-179.

[7] D. Giannakopoulou, and J. Magee, "Fluent model checking for event-based systems," Proc. of the 9th European Software Engineering Conf. (ESEC 2003) and the 11th ACM SIGSOFT Int'l Symposium on Foundations of Software Engineering (FSE 2003), ACM Press, 2003, pp. 257-266.

[8] Interaction Design Pattern (from Wikipedia), http://en.wikipedia.org/wiki/Interaction_design_pattern.

[9] ISDL home, http://isdl.ctit.utwente.nl.

[10] V. Jones, A. Rensink, T. Ruys, E. Brinksma, and A. van Halteren, "A formal MDA approach for mobile health systems," Proc. of the Second European Workshop on Model Driven Architecture (MDA) with an emphasis on Methodologies and Transformations (EWMDA 2004), Computing Laboratory, Kent CT2 7NF, Canterbury, UK, pp 28-35.

[11] Live Contacts home, http://livecontacts.telin.nl.

[12] medini QVT: ikv++ technologies home, http://www.ikv.de.

[13] Object Management Group, “MDA guide, V1.0.1,” omg/03-06-01, Jun. 2003.

[14] D. Quartel, T. Dirgahayu, and M. van Sinderen, "Model-driven design, simulation and implementation of service compositions in COSMO,” Int'l J. of Business Process Integration and Management, in press.

[15] G.H. Ter Hofte, R.A.A. Otte, H.C.J. Kruse, and M. Snijders, "Context-aware communication with Live Contacts", Conf Supplement of Computer Supported Cooperative Work (CSCW2004), Chicago, USA, Nov. 2004.

[16] S. Uchitel, G. Brunet, and M. Chechick, "Behavior model synthesis from properties and scenarios," Proc. of the 29th Int'l Conf. on Software Engineering (ICSE 2007), IEEE Computer Society Press, 2007, pp. 34-43.

[17] S. Uchitel, J. Kramer, and J. Magee, "Incremental elaboration of scenario-based specifications and behavior models using implied scenarios,” ACM Trans. on Software Engineering and Methodology, vol. 13, no 1, ACM Press, Jan. 2004, pp 37-85.

[18] Object Management Group, "OMG Model Driven Architecture: How systems will be built," available at www.omg.org/mda

[19] A. McNeile, and N. Simons, "Methods of behavior modeling: A commentary on behavior modeling techniques for MDA," unpublished, http://www.metamaxim.com/download/documents/Methods.pdf. 